\title{
Systematic Assessment of Coronary Calcium Detectability and Quantification on Four Generations of CT Reconstruction Techniques: a Patient and Phantom Study
}

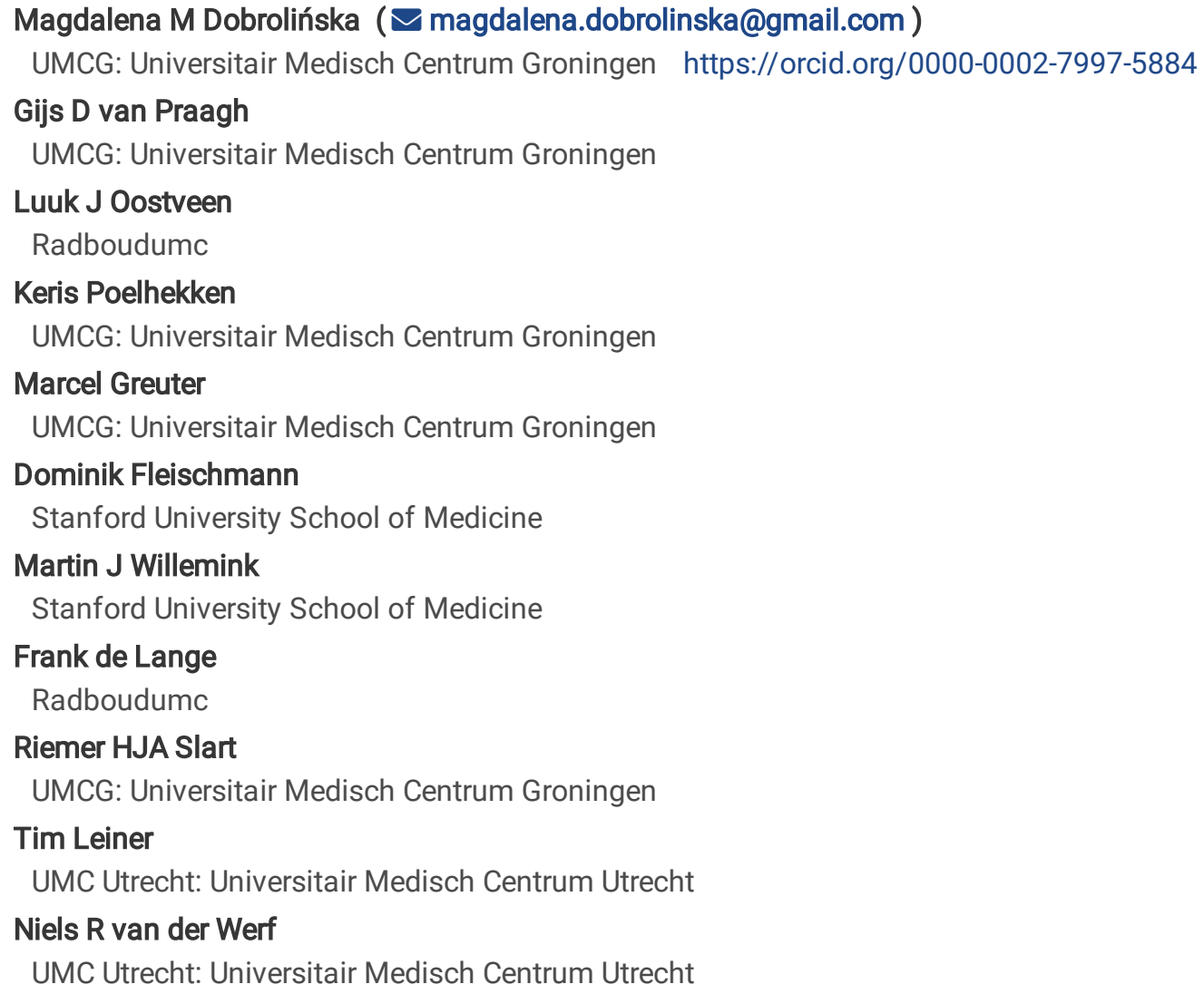

\section{Research Article}

Keywords: Deep learning, Phantoms, Imaging, Tomography, X-ray computed, Calcification, Radiation dosage, Image reconstruction Posted Date: February 17th, 2022

DOI: https://doi.org/10.21203/rs.3.rs-1343878/v1

License: (1) This work is licensed under a Creative Commons Attribution 4.0 International License. Read Full License 


\section{Abstract}

Purpose: In computed tomography, coronary artery calcium (CAC) scores are influenced by image reconstruction. For a newly introduced deep learning-based reconstruction (DLR), the effect on CAC scoring in relation to other algorithms is unknown. The aim of this study was to evaluate the effect of four generations of image reconstruction techniques (filtered back projection (FBP), hybrid iterative reconstruction (HIR), model-based iterative reconstruction (MBIR), and DLR) on CAC detectability, quantification, and risk classification.

Methods: First, CAC detectability was assessed with a dedicated static phantom containing 100 small calcifications varying in size and density. Second, CAC quantification was assessed with a dynamic coronary phantom with velocities equivalent to heart rates of 60-75 bpm. Both phantoms were scanned and reconstructed with four techniques. Last, scans of fifty patients were included and the Agatston calcium score was calculated for all four reconstruction techniques. FBP was used as a reference.

Results: In the phantom study, all reconstruction techniques resulted in less detected small calcifications for both, static and dynamic phantom. In the patient study, the cardiovascular risk classification resulted, for all reconstruction techniques, in excellent agreement with the reference, although MBIR resulted in significantly higher Agatston scores and $6 \%$ reclassification rate.

Conclusion: Agatston score agreement between FBP, HIR, and DLR was excellent, with a low-risk reclassification rate.

\section{Introduction}

Coronary artery calcium (CAC) is important for cardiovascular risk determination in asymptomatic individuals ${ }^{1}$. CAC is visualized with cardiac computed tomography (CT) and quantified using the Agatston score ${ }^{2}$. Furthermore, an Agatston score of zero is proven to be a strong negative predictor of future cardiovascular events ${ }^{3}$. This, in turn, indicates the importance of accurate detection and subsequent quantification of small calcified lesions.

One important factor influencing CAC quantification is the type of image reconstruction used in $\mathrm{CT}^{4}$. Over the last decade advanced reconstruction techniques such as hybrid iterative reconstruction (HIR) and model-based iterative reconstruction (MBIR) became available for $\mathrm{CT}^{5}$. These reconstruction algorithms reduce image noise, and therefore allow for a decrease in radiation dose while maintaining image quality equal to traditional filtered back projection (FBP) ${ }^{6,7}$. Previous studies have shown a good agreement in Agatston scores between FBP and HIR and MBIR ${ }^{8-10}$. However, it was also shown that HIR resulted in decreased Agatston scores for small and/or low density lesions ${ }^{9}$. Similarly, MBIR resulted in decreased detection of small calcifications ${ }^{8}$.

Recently, one of the main CT manufacturers introduced a new deep learning-based reconstruction (DLR) technique. DLR improves image quality by applying a deep learning network trained on pairs of high-dose, advanced MBIR and HIR images ${ }^{11}$ and prevents image quality degradation and 'plastic-like' appearance of the image ${ }^{12}$. As previously shown with low dose acquisitions, DLR outperforms MBIR in terms of noise reduction which may potentially allow for further radiation dose reduction beyond current levels ${ }^{11,13}$. However, the influence of this novel image reconstruction technique on CAC detection and quantification is unknown.

As previously noted, the detection of CAC, resulting subsequently in zero or non-zero Agatston scores, is of utmost importance for correct risk stratification. Because small or low-density CAC can resemble image noise and HIR, MBIR, and DLR all decrease image noise, these CT reconstruction techniques may impact the detection of very small or low-density CAC. This is even more important for acquisitions at a reduced radiation dose ${ }^{14}$. As previously shown, risk classification was underestimated up to $50 \%$ for CAC scores from IR images acquired at reduced radiation dose ${ }^{4}$. Consequently, the Society of Cardiovascular Computed Tomography recommends further evaluation of reconstruction techniques before clinical implementation ${ }^{15}$. Therefore, we designed a phantom study in which we aimed to investigate the influence of four reconstruction methods (FBP, HIR, MBIR, and DLR) on static and dynamic CAC detectability and quantification for standard and reduced radiation dose. Subsequently, we verified the effect of all four image reconstruction techniques on CAC quantification and risk classification in a patient study.

\section{Materials And Methods}

\subsection{Phantom study}


In this study, a small patient (300x200 mm) anthropomorphic thorax phantom (Thorax, QRM, Möhrendorf, Germany) was used (Fig. 1) ${ }^{16}$. To simulate large patient dimensions, an extension ring (Extension ring, QRM, Möhrendorf, Germany) of fat tissue equivalent material was used to increase the outer dimensions of the phantom to $400 \times 300 \mathrm{~mm}$.

CAC detectability was assessed with a cylindrical insert (D100, QRM, Möhrendorf, Germany). This insert contained one hundred small cylindrical calcifications differing in size ( 0.5 to $2.0 \mathrm{~mm}$ in diameter and length) and density (90 to $540 \mathrm{mg}$ hydroxyapatite $\left.(\mathrm{HA}) / \mathrm{cm}^{3}\right)^{17}$.

CAC quantification was assessed with the use of a dynamic artificial coronary artery, which was translated by a computer-controlled lever (Sim2D, QRM, Möhrendorf, Germany) in a water-filled compartment in the thorax phantom (Fig. 1). During acquisition, the artery remained static or moved at a constant velocity of $20 \mathrm{~mm} / \mathrm{s}$ in the horizontal plane during the scan phase, simulating a heart rate of 0 or 60-75 bpm, respectively ${ }^{18,19}$. Two arteries were used containing three cylindrical calcifications with equal dimensions (diameter: 5 $\mathrm{mm}$, length: $10 \mathrm{~mm}$ ), but varying densities of $196 \pm 3,408 \pm 2$ and $800 \pm 2 \mathrm{mgHA} / \mathrm{cm}^{3}$, designated as low, medium, and high density, respectively (Fig. 1).

\section{Data acquisition}

Both phantom sizes were scanned on a state-of-the-art 320 slice CT system (Aquilion One PRISM edition, Canon Medical Systems, Otawara, Japan) with routinely used clinical CAC protocols (Table 1). Automatic tube current selection (SureExposure 3D, Canon Medical Systems, Otawara, Japan) was used to select appropriate radiation dose levels for the small and large phantom size. The reference level was based on setting the automatic tube current modulation to a standard deviation (SD) of 27.76 at $3 \mathrm{~mm}$, with 40 and $300 \mathrm{~mA}$ as the minimum and maximum tube current, respectively. Next, tube current was reduced to $75 \%, 50 \%$, and $25 \%$ of the clinical radiation dose. Raw data was acquired at $120 \mathrm{kVp}$. Besides raw data reconstruction with FBP, three other reconstruction methods were used: HIR (adaptive iterative dose reduction 3D; AIDR 3D enhanced), MBIR (forward projected model based iterative reconstruction solution; FIRST standard), and DLR (advanced intelligent clear-IQ engine; AiCE standard) (Table 1). Each protocol was repeated ten times for the static and five times for the dynamic phantom. A larger number of repetitions was used for the static phantom, as the small size of the calcifications $(\leq 2 \mathrm{~mm})$ was highly impacted by partial volume effects due to the $3 \mathrm{~mm}$ slice thickness. Between each scan the phantom was manually repositioned (approximately $2 \mathrm{~mm}$ translational and 2 degrees rotational) to assess interscan variability.

Table 1

CAC acquisition and reconstruction parameters for phantom and patient study

\begin{tabular}{|lll|}
\hline & Phantom study & Patient study \\
\hline Acquisition mode & Axial & Axial \\
\hline ECG-triggering & Prospective & Prospective \\
\hline Peak tube potential [kVp] & $120 / 100$ & 120 \\
\hline $\begin{array}{l}\text { Reference image noise } \\
\text { [HU] }\end{array}$ & 27.76 & 27.76 \\
\hline Rotation time [s] & 0.275 & 0.275 \\
\hline Field of view [mm] & $220 \times 220$ & Patient specific \\
\hline Matrix size [pixels] & $512 \times 512$ & $512 \times 512$ \\
\hline $\begin{array}{l}\text { Slice thickness / } \\
\text { increment [mm] }\end{array}$ & $3.0 / 3.0$ & $3.0 / 3.0$ \\
\hline Reconstruction kernel & FC12 & FC12 \\
\hline Reconstruction algorithm & $\begin{array}{l}\text { FBP / AIDR 3D enhanced / FIRST standard / } \\
\text { AiCE standard }\end{array}$ & $\begin{array}{l}\text { FBP / AIDR 3D enhanced / FIRST standard / } \\
\text { AiCE standard }\end{array}$ \\
\hline ECG = electrocardiogram; bpm = beats per minute; FBP = filtered back projection. & \\
\hline
\end{tabular}


CAC detection and Agatston score calculations on the phantom scans were performed using a validated fully automated quantification method with vendor specific CAC scoring parameters ${ }^{20}$. A standard CAC scoring threshold of 130 Hounsfield units (HU) was used ${ }^{2}$.

For each scan, a background Agatston score (BAS) was calculated on a homogeneous part of the phantom, as described previously by Booij et al ${ }^{21}$. For scans with a nonzero BAS, it was unknown if a CAC was detected or if the score was based on noise only. For CAC detection purposes, a scan with a nonzero BAS was therefore defined as non-diagnostic and was omitted from further analysis.

\subsection{Patient study}

A patient study was performed to assess differences in Agatston scores resulting from the application of different reconstruction algorithms. This retrospective study was approved by the local ethics committee (CMO 2016-3045, Project 20045), who waived the requirement for patient informed consent after de-identification of all patient information from the study data. Raw data was acquired on the same CT system as used for the phantom scans, in a consecutive cohort of 50 patients between July and October 2020 (Table 2). All patients were scanned at $120 \mathrm{kVp}$. Raw data was reconstructed using the same four reconstruction methods as for the phantom studies: FBP, HIR, MBIR, and DLR.

Table 2

Patients' characteristics of the 50 patients included in the study.

\begin{tabular}{|ll|}
\hline Patients' characteristics & Patient study \\
\hline Median age (range) [years] & $60(41-77)$ \\
\hline Female & $32(64 \%)$ \\
\hline Heart rate (range) [bpm] & $60(57-68)^{\star}$ \\
\hline Median Agatston score (range) & $61(0-2935)$ \\
\hline *For 2 patients, heart rate could not be retrieved retrospectively \\
\hline
\end{tabular}

Agatston scores in patient scans were determined using a dedicated workstation (Vitrea 7.11; Vital Images Inc.).

\subsection{Statistical analysis}

Agatston scores resulting from the default clinical protocol (120 kVp, 100\% dose, FBP) were used as the reference for both the phantom and patient study. Scores from other acquisition and reconstruction settings were compared with this reference. For the phantom study, the comparison was performed within the same repetition. For the different combinations of radiation dose, and reconstruction method, deviations of more than $10 \%$ in Agatston score from the reference were considered clinically relevant ${ }^{22}$. Categorical variables and number of detected calcifications were presented as percentages. For the static phantom experiments, a false-positive result was defined as a calcification not detected on the reference scan, a false-negative result was defined as calcification detected on the reference scan but not on the HIR, MBIR, or DLR scan. Depending on the distribution of the data, continuous variables were presented as means with standard deviation $( \pm S D)$ or medians with interquartile region (IQR, $1 \mathrm{st}-3 \mathrm{rd})$.

Patient Agatston scores resulting from the different reconstruction techniques were compared with the reference score (120 kVp, FBP) using Bonferroni corrected Wilcoxon signed-rank tests. Next, patients were divided into five risk groups (0 Agatston score $-0 ; 0.1$ to 10 Agatston score $-1 ; 10.1$ to 100 Agatston score - 2; 100.1 to 400 Agatston score $-3 ;>400$ Agatston score -4 ) and the agreement in risk classification between the different reconstruction methods was compared based on a Cohen weighted linear $\mathrm{K}$ with $95 \%$ confidence intervals $(95 \% \mathrm{Cl})$. The cardiac risk classification was determined for each patient and each reconstruction technique ${ }^{23}$. The agreement between FBP Agatston score and HIR, MBIR, and DLR Agatston score was analysed with Bland-Altman plots.

P values smaller than 0.05 were considered statistically significant. SPSS version 25 (IBM Corp., Armonk, NY, USA) was used for statistical analyses.

\section{Results}

\subsection{Phantom study}


Full dose settings resulted in 80 and $300 \mathrm{~mA}$ for the small and large phantom, respectively. Tube currents were reduced to the nearest available setting to obtain $75 \%, 50 \%$, and $25 \%$ of the full dose setting. The resulting volume CT dose indexes (CTDI vol $)$ for $100 \%$ dose setting were $1.2 \mathrm{mGy}(120 \mathrm{kVp})$ for the small phantom and $4.4 \mathrm{mGy}(120 \mathrm{kVp})$ for the large phantom.

\subsubsection{CAC detectability}

For all used reconstruction algorithms, the CT numbers for a calcification with a density of $300 \mathrm{mgHA} / \mathrm{cm}^{3}$ and varying sizes within the small phantom are depicted in Fig. 2. This figure shows a difference in the HU peak reached by each of the reconstruction methods, whereby the CAC scoring threshold of $130 \mathrm{HU}$ is not reached for the smallest calcification by MBIR and DLR.

For all repeated scans, the reference protocol resulted in a CAC detection of 150 and 87 calcifications out of 1000 for the small and large phantom, respectively. Relative results for the other reconstruction algorithms and dose levels are shown in Fig. 3 and supplementary Figure S1.

For the small phantom at full dose, MBIR, and DLR resulted in $4 \%$, and $1 \%$ less detected calcifications, while $8 \%$ more calcifications were detected with HIR (Figure S1). For the large phantom at full dose, $2 \%, 22 \%$, and $9 \%$ less calcifications were detected for HIR, MBIR, and DLR, respectively (Fig. 3).

For the small phantom, $75 \%$ dose with $120 \mathrm{kVp}$ resulted in $7 \%, 2 \%, 55 \%$, and $59 \%$ less detected calcifications for FBP, HIR, MBIR, and DLR, respectively. In the large phantom the reduction was even larger, with $10 \%, 18 \%, 84 \%$, and $80 \%$ less detected calcifications, respectively. The number of missed calcifications was even more pronounced for $50 \%$ and $25 \%$ dose (Fig. 3 and supplementary Figure S1).

\subsubsection{CAC quantification}

For the small static phantom, median (IQR) Agatston scores were 96 (95-108), 350 (344-363), and 413 (403-427) for the low-, medium-, and high-density CAC in the reference protocol. At 60-75 bpm, these Agatston scores changed to 87 (82-88), 379 (368419 ), and 474 (464-513) (Supplementary Figure S2). This resulted in the overall change of Agatston score by $-22 \%, 9 \%$, and $25 \%$ for low, medium, and high-density calcifications, respectively (Supplementary Figure S2).

For the large static phantom, Agatston scores were 74 (70-82), 303 (301-306), and 381 (379-388) for the low-, medium-, and highdensity CAC (Fig. 4). These Agatston scores changed at 60-75 bpm to 48 (42-67), 355 (348-361), and 503 (469-515). Briefly, for the large phantom Agatston scores increased compared to the static situation by $10.4 \%(-49-115.2 \%), 200 \%$ (103.2-346\%), and $189.5 \%$ (120.3-400.6\%) for the low-, medium-, and high- density calcifications, respectively (Fig. 4).

As compared to reference Agatston scores, deviations in Agatston scores for data reconstructed with the other reconstruction methods, were non-relevant (< 10\%) (Fig. 4 and Supplementary Figure S2). For 120 kVp with 50\% radiation dose, most reconstruction methods resulted in small non-relevant deviations in Agatston score, as depicted on Fig. 4.

\subsection{Patient study}

The age range of the 50 patients was 41-77 years with a median age of 60 years and 32 (64\%) patients were female. Median dose length product for the calcium scoring acquisitions was $60.2 \mathrm{mGycm}$ (full range: $30.8-73.6 \mathrm{mGycm}$ ) corresponding to an estimated effective dose of $1.56(0.8-1.91) \mathrm{mSv}$ using a conversion factor of $0.026 \mathrm{mSv} / \mathrm{mGycm}^{24}$.

\subsubsection{CAC quantification and detectability}

The median (IQR) Agatston scores were 61 (5.5-435.0), 63 (8.5-412.0), 81.5 (9.25-435.0), and 72.5 (9.25-401.0), for FBP, HIR, MBIR, and DLR, respectively. Only MBIR Agatston scores were significantly different from FBP $(p<0.001)$. Within all reconstruction methods, only for MBIR one false-positive calcification was detected. Additionally, differences in Agatston score between FBP and HIR, MBIR, and DLR, increased with increasing Agatston scores (Fig. 5). The difference between the four reconstruction methods in calcium detection is depicted on Fig. 6.

\subsubsection{Risk classification}


Overall, the agreement between cardiovascular risk classification based on FBP compared to HIR, MBIR, and DLR was excellent ( $\mathrm{K}=$ 0.97, 95\% Cl: 0.94-1.0; $\mathrm{k}=0.96,95 \% \mathrm{Cl}: 0.92-1.0 ; \mathrm{k}=0.97,95 \% \mathrm{Cl}: 0.94-1.0$ ) (Table 3a-c). However, based on MBIR, three patients $(6 \%)$ were included in a higher risk category as compared to FBP. Within these patients, one was reclassified from zero to a non-zero Agatston score. For HIR as well as for DLR, reclassification occurred in two cases (4\%?) (Table 3a-c). In both reconstruction methods one case to a lower category and one to a higher category.

Table3: a-c. The agreement between patient risk classification based on FBP and risk classification based on MBIR, HIR, and DLR respectively.

a)

Risk classification based on FBP

\begin{tabular}{llllllll}
\hline & $\mathbf{0}$ & $\mathbf{1}$ & $\mathbf{2}$ & $\mathbf{3}$ & $\mathbf{4}$ & \\
\hline Risk classification based on MBIR & $\mathbf{0}$ & 10 & 0 & 0 & 0 & 0 & $10(20 \%)$ \\
\hline & $\mathbf{1}$ & 1 & 1 & 0 & 0 & 0 & $2(4 \%)$ \\
\hline $\mathbf{2}$ & 0 & 1 & 16 & 0 & 0 & $17(34 \%)$ \\
\hline $\mathbf{3}$ & 0 & 0 & 1 & 7 & 0 & $8(16 \%)$ \\
\hline $\mathbf{4}$ & 0 & 0 & 0 & 0 & 13 & $13(26 \%)$ \\
\hline & $11(22 \%)$ & $2(4 \%)$ & $17(34 \%)$ & $7(14 \%)$ & $13(26 \%)$ & 50
\end{tabular}

b)

\begin{tabular}{llllllll}
\multicolumn{7}{c}{ Risk classification based on FBP } \\
\hline Risk classification based on HIR & $\mathbf{0}$ & $\mathbf{1}$ & $\mathbf{2}$ & $\mathbf{3}$ & $\mathbf{4}$ & \\
\hline & $\mathbf{0}$ & 11 & 0 & 0 & 0 & 0 & $11(22 \%)$ \\
\hline $\mathbf{1}$ & 0 & 1 & 0 & 0 & 0 & $1(2 \%)$ \\
\hline $\mathbf{2}$ & 0 & 1 & 17 & 0 & 0 & $18(36 \%)$ \\
\hline $\mathbf{3}$ & 0 & 0 & 0 & 7 & 1 & $8(16 \%)$ \\
\hline $\mathbf{4}$ & 0 & 0 & 0 & 0 & 12 & $12(24 \%)$ \\
\hline & $11(22 \%)$ & $2(4 \%)$ & $17(34 \%)$ & $7(14 \%)$ & $13(26 \%)$ & 50
\end{tabular}

c)

\begin{tabular}{llllllll}
\multicolumn{7}{c}{ Risk classification based on FBP } \\
\hline Risk classification based on DLR & $\mathbf{0}$ & $\mathbf{1}$ & $\mathbf{2}$ & $\mathbf{3}$ & $\mathbf{4}$ & \\
\hline & $\mathbf{0}$ & 11 & 0 & 0 & 0 & 0 & $11(22 \%)$ \\
\hline & $\mathbf{1}$ & 0 & 1 & 0 & 0 & 0 & $1(2 \%)$ \\
\hline $\mathbf{2}$ & 0 & 1 & 17 & 0 & 0 & $18(36 \%)$ \\
\hline $\mathbf{3}$ & 0 & 0 & 0 & 7 & 1 & $8(16 \%)$ \\
\hline $\mathbf{4}$ & 0 & 0 & 0 & 0 & 12 & $12(24 \%)$ \\
\hline & $11(22 \%)$ & $2(4 \%)$ & $17(34 \%)$ & $7(14 \%)$ & $13(26 \%)$ & 50
\end{tabular}

Risk groups are defined as follows: 0 Agatston score - 0;0.1 to 10 Agatston score - 1; 10.1 to 100 Agatston score $-2 ; 100.1$ to 400 Agatston score - 3; $>400$ Agatston score -4 . 


\section{Discussion}

The main finding of the phantom part in the present study is that detection of small calcifications at routine (100\%) radiation dose is reduced up to $22 \%$ depending on the used reconstruction algorithm. Furthermore, this trend was even more pronounced on reduced radiation dose scans. For CAC quantification, our dynamic phantom study showed no clinically relevant differences in Agatston score based on reconstruction algorithm for the routine radiation dose protocol. The patient study showed excellent agreement between FBP and HIR, MBIR, and DLR, with only a small number of risk reclassifications, although MBIR resulted in significantly higher Agatston scores.

To the best knowledge of the authors, this study is the first to systematically assess the influence of all reconstruction techniques currently available for one vendor on CAC detection and quantification. Compared to FBP all reconstruction methods reduced CAC detection, except in the case of the small chest phantom at full dose level. Both IR techniques as well as DLR reduce image noise ${ }^{11}$. The, in general, reduced CAC detectability in comparison with FBP for these reconstruction techniques might therefore be explained by erroneous identification of CAC containing voxels as noise. Furthermore, as we presented in this study, decreased detectability may be due to reduced $\mathrm{HU}$ peaks in small calcifications. This behavior will, of course, be more pronounced at reduced tube current and increased patient size, where noise levels are increased. As a result, HIR, but especially MBIR and DLR may miss small calcifications and improperly classify patients into the zero Agatston score risk group. However, based on our patient study, none of the patients was incorrectly assigned to the zero Agatston score group.

Independent of the reconstruction method, for medium and large density calcifications, the Agatston score increased with velocity, while for small density calcification, Agatston score decreased. This finding is in line with previous results of van der Werf et al. ${ }^{19}$ and Groen et al. ${ }^{25}$ and might be explained by motion blurring. Due to motion blurring, the number of voxels above $130 \mathrm{HU}$ increases in medium and large density calcifications, which increases the Agatston score. In low density calcifications, in turn, the number of voxels above $130 \mathrm{HU}$ decreases, which decreases the Agatston score.

As we know from the CONFIRM registry, small calcifications visually detected on CCTA scans in patients previously assigned to the zero Agatston score risk group, increased risk of major adverse cardiac events ${ }^{26}$. Therefore, detectability of small calcifications plays a crucial role in further patient management. Importantly, when reduced tube currents were used, detectability of small calcifications decreased, especially for MBIR and DLR. Our hypothesis is that this can be explained by the need for increased noise suppression by these reconstruction algorithms. Therefore, based on these static phantom results we assume that patients might be misclassified into the zero Agatston score risk group when a reduced radiation dose protocol is used. Future patient studies with more small calcifications should verify this.

Additionally, at routine tube current level, the current study did not show relevant differences between reconstruction methods in terms of Agatston scores. However, when the tube current was decreased to $50 \%$, Agatston score of low density calcifications acquired from the large dynamic phantom deviated from the standard measurement ${ }^{2}$. Therefore, as also underlined in SCCT guidelines ${ }^{15}$, caution should be taken in terms of radiation dose reduction by decreasing tube current, especially in combination with iterative reconstruction methods.

The patient study showed that only the Agatston score measured from MBIR differed significantly from the reference Agatston score based on FBP. When considering patients with a zero Agatston score as defined by the reference method, MBIR classified one patient as a nonzero Agatston score, thereby increasing the risk classification. However, similar results were presented before, with $17 \%$ of cases reclassified into higher risk group, including $8 \%$ of patient misclassified as non-zero Agatston scores ${ }^{8}$. One explanation for this behavior might be the impact of the edge enhancement algorithm, whereby more pronounced CAC edges increase overall Agatston scores. Also, the Bland-Altman limits of agreement of MBIR compared with FBP were almost twice as large as the limits of HIR or DLR compared with FBP. Nevertheless, overall agreement in risk classification was excellent for all reconstruction methods. Importantly, for our patient group, none of the patients was reclassified as a false negative. Currently, both American and European guidelines use CAC scoring as an additional tool not only for patient risk classification, but also for guiding statin and aspirin therapy ${ }^{27}$. Therefore, the lack of CAC measurement reproducibility and its dependency on different reconstruction methods, may affect patient management and outcome ${ }^{23}$. Based on patients results from our study and using FBP as reference, the most accurate calcium scoring was achieved when HIR or DLR was used, in terms of correct patient risk classification. 
This study has several limitations. First, while our systematic analysis included both a static and dynamic phantom as well as a patient study, we only included a small number of patients. Moreover, only twelve patients (24\%) presented with Agatston score between 0 and 10, which is the most susceptible group in terms of calcium detectability. Despite the relatively small sample size of the patient study $(n=50)$, we feel that the size was large enough for validation purposes of our phantom study. Second, we acquired data from one vendor. Therefore, a multivendor study analyzing the influence of different reconstruction methods on calcium detectability, quantification and risk stratification is certainly needed. Third, all patients were scanned with the standard protocol. Therefore, the effect of decreased radiation dose could not be evaluated in patients. Fourth, the D100 phantom is a static phantom. Thus, we were not able to acquire dynamic detectability phantom data. However, due to the decrease in detectability, even in a static situation, care should be taken when using non-FBP reconstructions for detecting CAC with this CT system.

\section{Conclusions}

In conclusion, based on our patient results, HIR and DLR reconstructed scans resulted in similar Agatston scores with excellent agreement and low-risk reclassification rate compared with routine reconstructed scans (FBP). These results suggest that these reconstruction methods might be applied for CAC scoring. However, based on our phantom study, caution should be taken when patients have Agatston scores between 0 and 10, as detectability of small calcifications varies with the used reconstruction algorithm, especially with MBIR and DLR. More clinical studies with a large amount of low Agatston score calcifications are needed to verify this. Moreover, decreased radiation dose impaired Agatston scoring of small calcifications which may lead to improper patient risk classification.

\section{Declarations}

Conflicts of interests: None

Disclosure: The authors have nothing to disclose.

Funding: M.D. received the "EACVI Research Grant 2020" and "Specialised Research Fellowship 2019 Grant from "Club 30" and Polish Cardiac Society";

\section{Authors contribution:}

Conceptualization, Niels van der Werf; methodology, M Dobrolinska., GD van Praagh, LJ Oostveen, MJW Greuter, M.J.W., Niels van der Werf.; software, Niels van der Werf., ; formal analysis, M Dobrolinska, GD van Praagh, LJ Oostveen, Niels van der Werf ; data curation, M Dobrolinska, GD van Praagh, LJ Oostveen, MJW Greuter, MJ Willemink, Niels van der Werf; writing-original draft preparation, M Dobrolinska, GD van Praagh, LJ Oostveen, Niels van der Werf.; writing-review and editing, K Poelhekken, MJW Greuter, D Fleischmann, MJ Willemink, F de Lange, RHJA Slart, T Leiner;; visualization, GD van Praagh, Niels van der Werf, LJ Oostveen, M Dobrolinska, supervision, Niels van der Werf. All authors have read and agreed to the published version of the manuscript.

\section{Ethics approval:}

Authors confirm that study was approved by the local ethics committee (CMO 2016-3045, Project 20045), who waived the requirement for patient informed consent after de-identification of all patient information from the study data.

\section{References}

1. Knuuti J, Wijns W, Achenbach S et al (2020) 2019 ESC guidelines for the diagnosis and management of chronic coronary syndromes. Eur Heart J 41(3):407-477. doi:10.1093/eurheartj/ehz425

2. Agatston AS, Janowitz WR, Hildner FJ, Zusmer NR, Viamonte M, Detrano R (1990) Quantification of coronary artery calcium using ultrafast computed tomography. J Am Coll Cardiol 15(4):827-832. doi:10.1016/0735-1097(90)90282-T

3. Blaha MJ, Cainzos-Achirica M, Greenland P et al (2016) Role of Coronary Artery Calcium Score of Zero and Other Negative Risk Markers for Cardiovascular Disease: the Multi-Ethnic Study of Atherosclerosis (MESA). Circulation.

doi:10.1161/CIRCULATIONAHA.115.018524

Page 8/14 
4. Kurata A, Dharampal A, Dedic A et al (2013) Impact of iterative reconstruction on CT coronary calcium quantification. Eur Radiol 23(12):3246-3252. doi:10.1007/s00330-013-3022-8

5. Willemink MJ, Noël PB (2019) The evolution of image reconstruction for CT-from filtered back projection to artificial intelligence. Eur Radiol 29(5):2185-2195. doi:10.1007/s00330-018-5810-7

6. Xu F, Mueller K (2007) Real-time 3D computed tomographic reconstruction using commodity graphics hardware. Phys Med Biol. doi:10.1088/0031-9155/52/12/006

7. Willemink MJ, De Jong PA, Leiner T et al (2013) Iterative reconstruction techniques for computed tomography Part 1: Technical principles. Eur Radiol. doi:10.1007/s00330-012-2765-y

8. Choi AD, Leifer ES, Yu JH et al (2019) Reduced radiation dose with model based iterative reconstruction coronary artery calcium scoring. Eur J Radiol 111:1-5. doi:10.1016/j.ejrad.2018.12.010

9. Tatsugami F, Higaki T, Fukumoto W et al (2015) Radiation dose reduction for coronary artery calcium scoring at 320-detector CT with adaptive iterative dose reduction 3D. Int J Cardiovasc Imaging. doi:10.1007/s10554-015-0637-7

10. Tang YC, Liu YC, Hsu MY, Tsai HY, Chen CM (2018) Adaptive Iterative Dose Reduction 3D Integrated with Automatic Tube Current Modulation for CT Coronary Artery Calcium Quantification: Comparison to Traditional Filtered Back Projection in an Anthropomorphic Phantom and Patients. Acad Radiol. doi:10.1016/j.acra.2017.12.018

11. Akagi M, Nakamura Y, Higaki T et al (2019) Deep learning reconstruction improves image quality of abdominal ultra-highresolution CT. Eur Radiol. doi:10.1007/s00330-019-06170-3

12. Geyer LL, Schoepf UJ, Meinel FG et al (2015) State of the Art: Iterative CT Reconstruction Techniques. Radiology 276(2):339-357. doi:10.1148/radiol.2015132766

13. Higaki T, Nakamura Y, Zhou J et al (2020) Deep Learning Reconstruction at CT: Phantom Study of the Image Characteristics. Acad Radiol. doi:10.1016/j.acra.2019.09.008

14. Hecht HS, Cronin P, Blaha MJ et al (2017) 2016 SCCT/STR guidelines for coronary artery calcium scoring of noncontrast noncardiac chest CT scans: A report of the Society of Cardiovascular Computed Tomography and Society of Thoracic Radiology. J Cardiovasc Comput Tomogr. doi:10.1016/j.jcct.2016.11.003

15. Hecht H, Blaha MJ, Berman DS et al (2017) Clinical indications for coronary artery calcium scoring in asymptomatic patients: Expert consensus statement from the Society of Cardiovascular Computed Tomography. J Cardiovasc Comput Tomogr. doi:10.1016/j.jcct.2017.02.010

16. McCollough CH, Ulzheimer S, Halliburton SS, Shanneik K, White RD, Kalender WA (2007) Coronary artery calcium: a multiinstitutional, multimanufacturer international standard for quantification at cardiac CT. Radiology 243(2):527-538. doi:10.1148/radiol.2432050808

17. Groen JM, Kofoed KF, Zacho M, Vliegenthart R, Willems TP, Greuter MJW (2013) Calcium score of small coronary calcifications on multidetector computed tomography: results from a static phantom study. Eur J Radiol 82(2):e58-63. doi:10.1016/j.ejrad.2012.09.018

18. Husmann L, Leschka S, Desbiolles $L$ et al (2007) Coronary artery motion and cardiac phases: Dependency on heart rate Implications for CT image reconstruction. Radiology 245(2):567-576. doi:10.1148/radiol.2451061791

19. van der Werf NR, Willemink MJ, Willems TP, Vliegenthart R, Greuter MJW, Leiner T (2018) Influence of heart rate on coronary calcium scores: a multi-manufacturer phantom study. Int J Cardiovasc Imaging 34(6):959-966. doi:10.1007/s10554-017-1293-x

20. van Praagh GD, van der Werf NR, Wang J et al (May 2021) Fully automated quantification method (FQM) of coronary calcium in an anthropomorphic phantom. Med Phys. doi:10.1002/mp.14912

21. Booij R, van der Werf NR, Budde RPJ, Bos D, van Straten M (2020) Dose reduction for CT coronary calcium scoring with a calciumaware image reconstruction technique: a phantom study. Eur Radiol. doi:10.1007/s00330-020-06709-9

22. van der Werf NR, Booij R, Schmidt B et al (May 2021) Evaluating a calcium-aware kernel for CT CAC scoring with varying surrounding materials and heart rates: a dynamic phantom study. Eur Radiol. doi:10.1007/s00330-021-08076-5

23. Detrano R, Guerci AD, Carr JJ et al (2008) Coronary Calcium as a Predictor of Coronary Events in Four Racial or Ethnic Groups. N Engl J Med. doi:10.1056/nejmoa072100

24. Trattner S, Halliburton S, Thompson CM et al (2018) Cardiac-Specific Conversion Factors to Estimate Radiation Effective Dose From Dose-Length Product in Computed Tomography. JACC Cardiovasc Imaging 11(1):64-74. doi:10.1016/j.jcmg.2017.06.006 
25. Groen JM, Greuter MJ, Schmidt B, Suess C, Vliegenthart R, Oudkerk M (2007) The Influence of Heart Rate, Slice Thickness, and Calcification Density on Calcium Scores Using 64-Slice Multidetector Computed Tomography: A Systematic Phantom Study.Invest Radiol. ; 42(12).

https://journals.Iww.com/investigativeradiology/Fulltext/2007/12000/The_Influence_of_Heart_Rate,_Slice_Thickness,_and.8.aspx

26. Han D, Klein E, Friedman J et al (2020) Prognostic significance of subtle coronary calcification in patients with zero coronary artery calcium score: From the CONFIRM registry. Atherosclerosis 309:33-38. doi:10.1016/j.atherosclerosis.2020.07.011

27. Osei AD, Mirbolouk M, Berman D et al (2021) Prognostic value of coronary artery calcium score, area, and density among individuals on statin therapy vs. non-users: The coronary artery calcium consortium. Atherosclerosis 316:79-83. doi:10.1016/j.atherosclerosis.2020.10.009

\section{Figures}
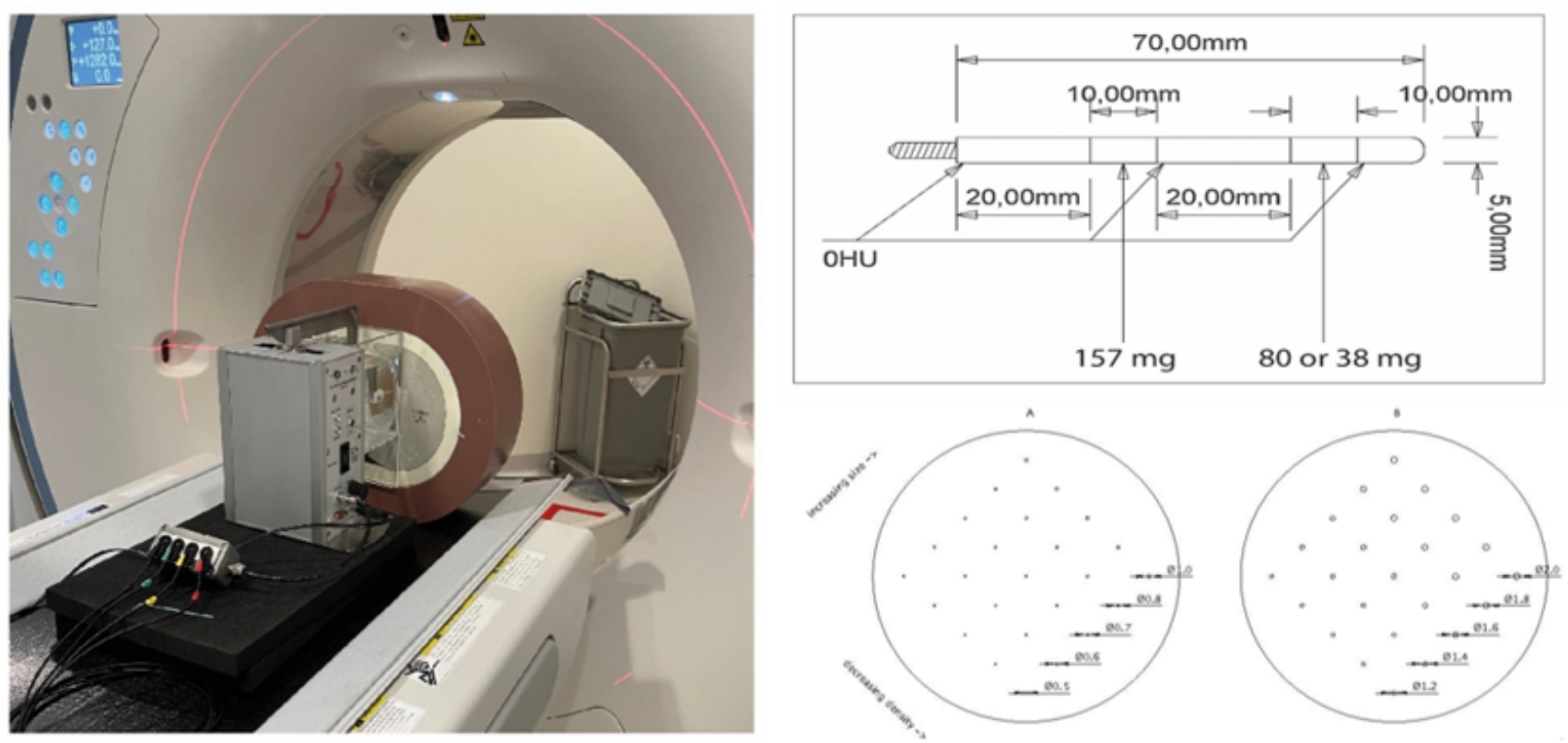

Figure 1

Overview of the phantom setup with the anthropomorphic thorax phantom and motion controller (left), and a schematic overview of the used calcium inserts for dynamic scan (upper right) and for static scan (bottom right). 


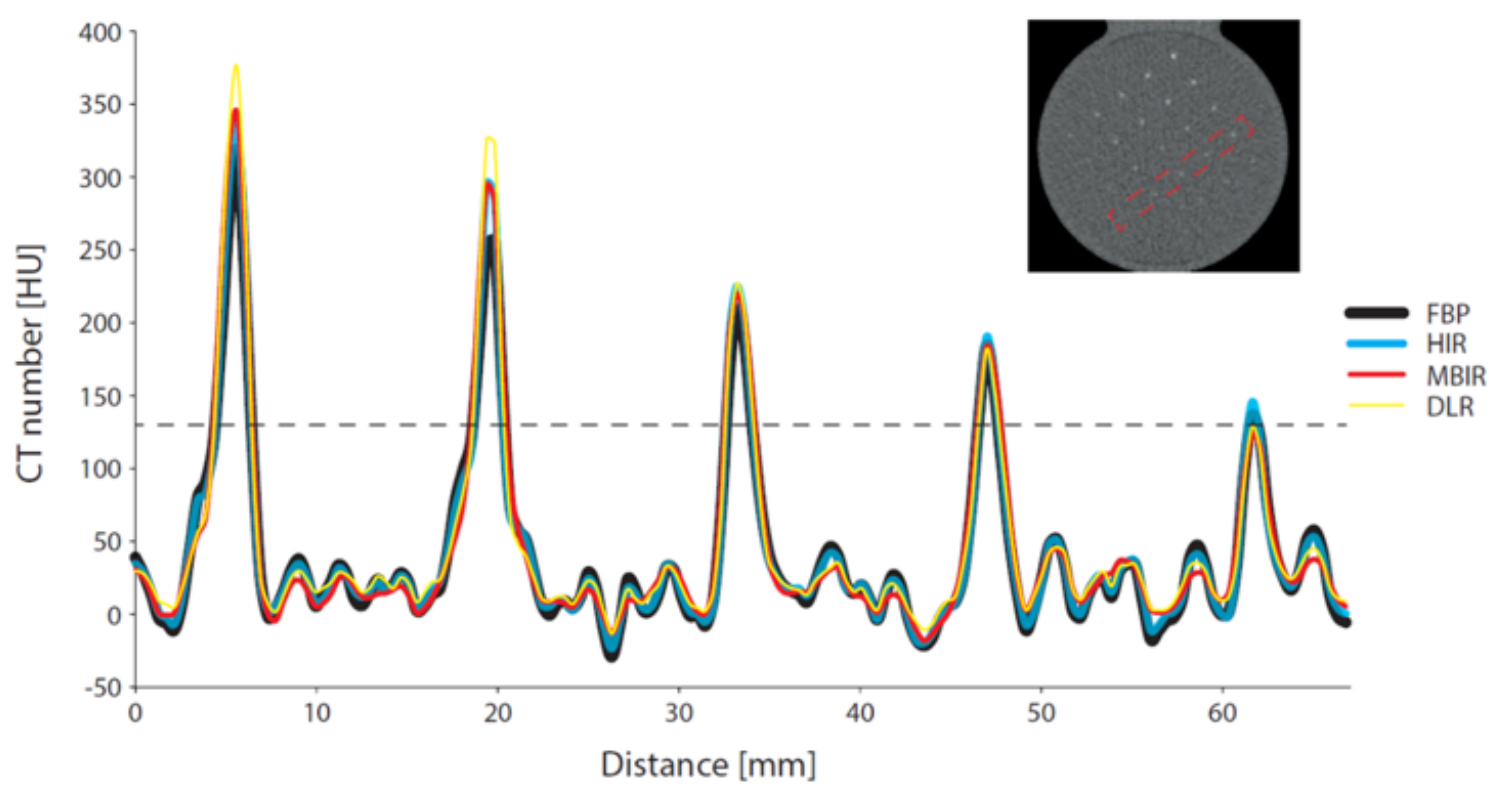

Figure 2

A profile plot through calcifications with $300 \mathrm{mgHA} / \mathrm{cm} 3$ of 2, 1.8, 1.6, 1.4 and $1.2 \mathrm{~mm}$ in diameter, respectively, as indicated in the red box in the right-upper image. These plots summarize the difference between the four reconstruction methods (FBP, HIR, MBIR, DLR). The conventional CAC scoring threshold of $130 \mathrm{HU}$ is indicated with a dotted line. As depicted on the plot, calcifications of the lowest diameter reconstructed with MBIR and DLR, do not reach the $130 \mathrm{HU}$ threshold.

FBP - filtered back projection, HIR - hybrid iterative reconstruction, MBIR - model-based iterative reconstruction, $D L R$ - deep learningbased reconstruction

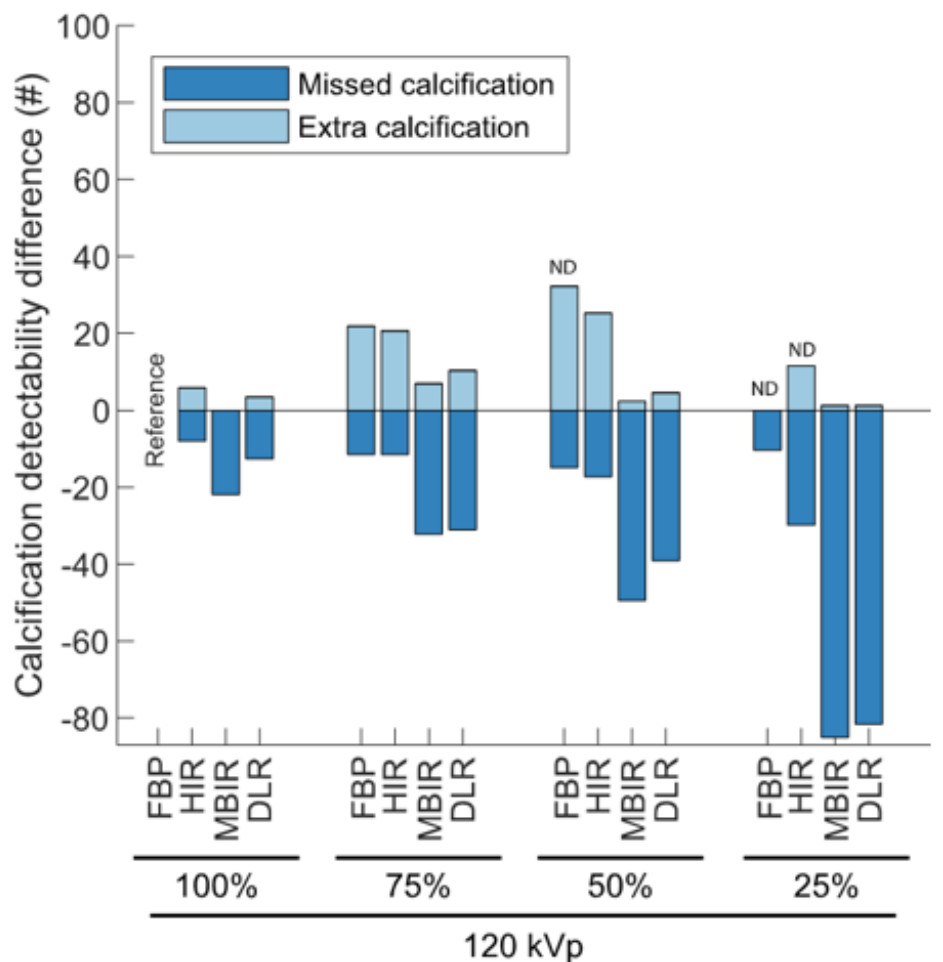

Page $11 / 14$ 


\section{Figure 3}

Difference in total number of detected calcifications of the static (D100) insert in the large thorax phantom for all combinations of tube currents (in percentage of reference) and reconstruction methods compared with the reference (120 kVp, 100\% dose, FBP). For each repetition, a calcification was defined as 'missed' when the calcification was detected with the reference protocol but was not detected with varying acquisition and/or reconstructions parameters. The opposite was defined as an 'extra calcification'. All repetitions with $B A S>0$ were defined as nondiagnostic (ND) image quality and were therefore omitted from the analysis.

$B A S$ - background Agatston score, FBP - filtered back projection, HIR - hybrid iterative reconstruction, MBIR - model-based iterative reconstruction, DLR - deep learning-based reconstruction, \# - number
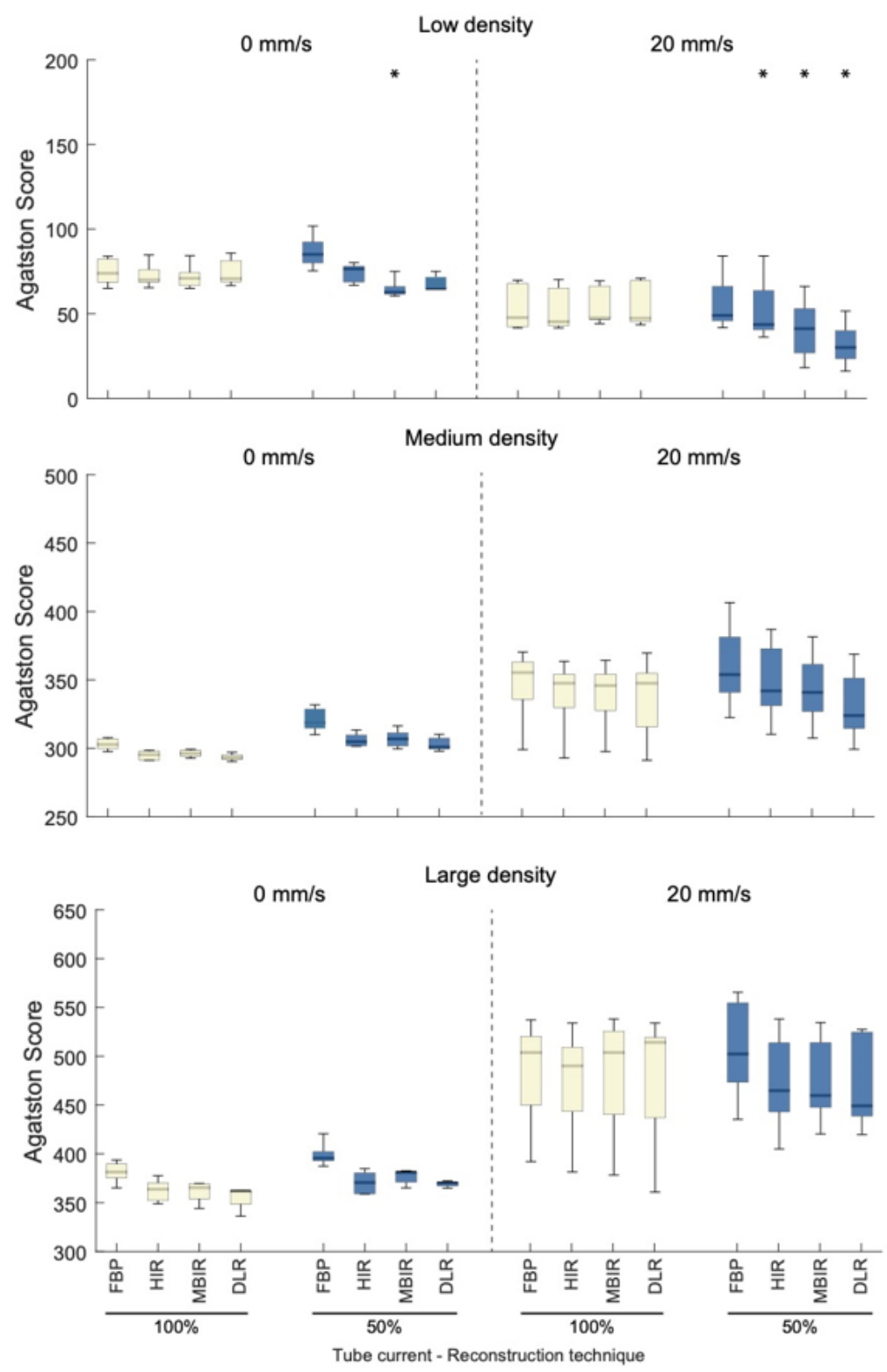

Figure 4

Large phantom static and dynamic Agatston scores for the low (top), medium (middle) and high (bottom) density calcifications, for all used tube current (in percentage of reference), and reconstruction methods. Asterix (*) marks a protocol that results in a clinically relevant (>10\% change) median Agatston score change compared with the reference protocol. 

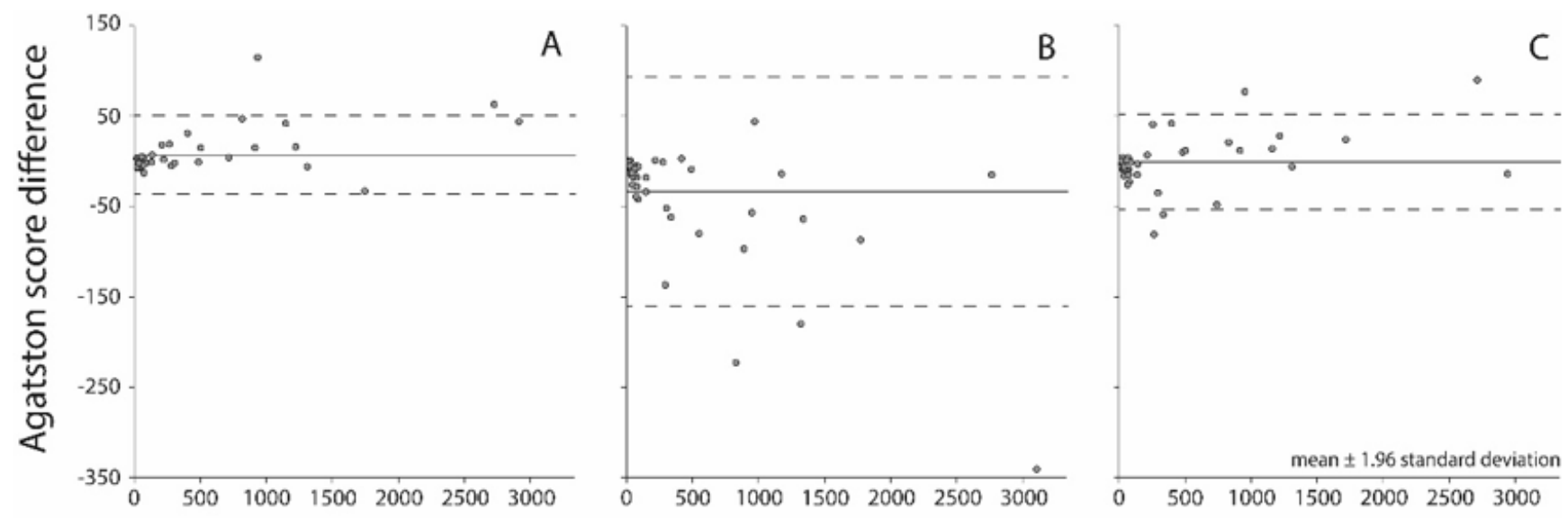

\section{Mean Agatston score}

\section{Figure 5}

Bland-Altman plots of the difference between HIR (A), MBIR (B) and DLR $(C)$ and FBP for all fifty patients. Agatston score difference was calculated as FBP Agatston score minus IR Agatston score. The solid line resembles a mean difference, the dashed lines resemble standard deviation.

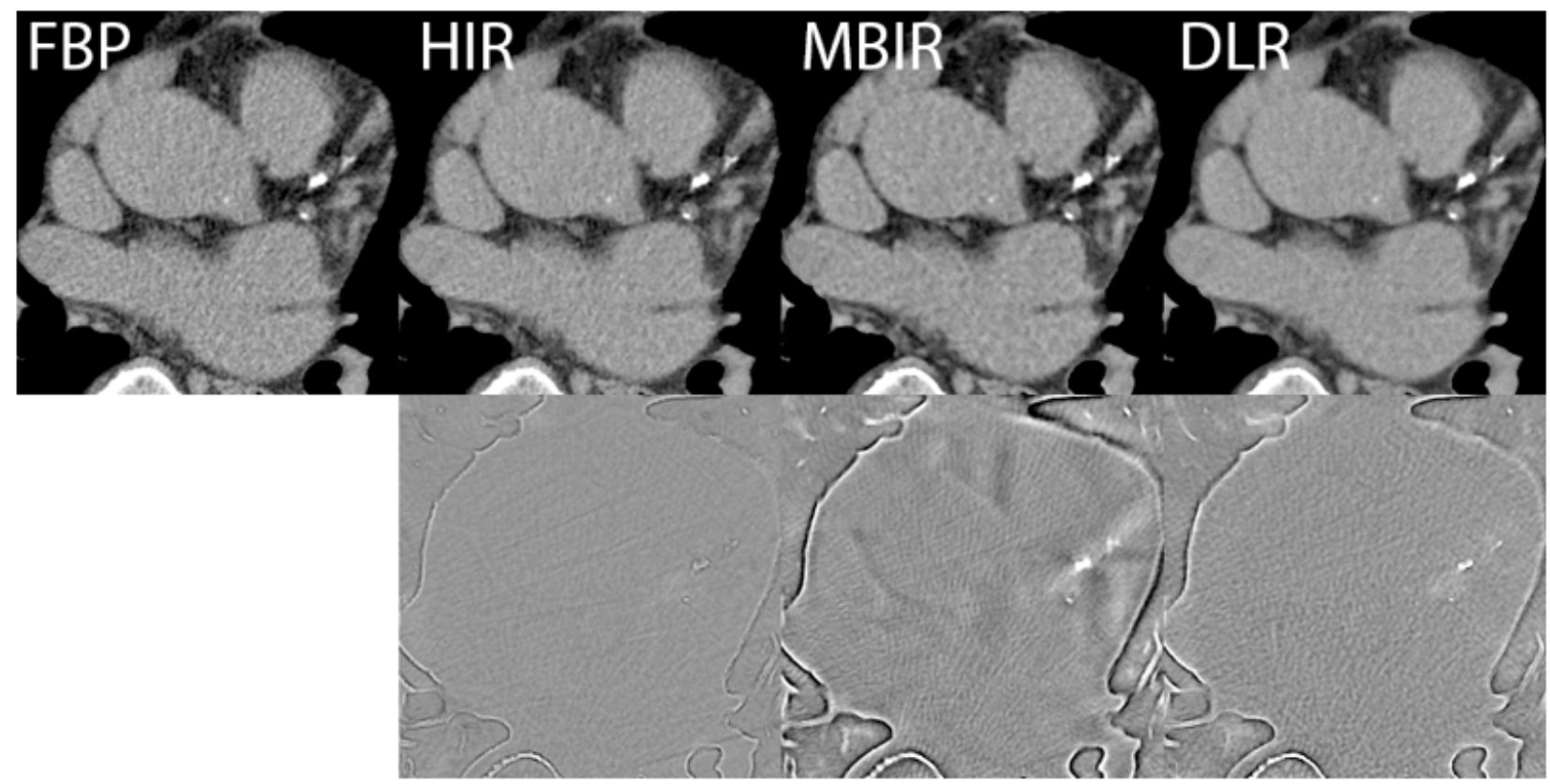

Figure 6

CAC detection for FBP, HIR, MBIR and DLR for one of the patients. Window width (WW) and window level (WL) setting were constant for the upper row: $W W=380, W L=40$. The bottom row shows subtraction images between FBP and HIR, MBIR and DLR. For MBIR, overall CAC quantification increases with respect to FBP.

FBP - filtered back projection, HIR - hybrid iterative reconstruction, MBIR - model-based iterative reconstruction, $D L R$ - deep learningbased reconstruction 


\section{Supplementary Files}

This is a list of supplementary files associated with this preprint. Click to download.

- Supplementaryfigure1.docx

- Supplementaryfigure2.docx 